\title{
Digital Technologies as a Panacea for Social Isolation and Loneliness among Older Adults: An Intervention Model for Flourishing and Wellbeing
}

Visual Technologies as a Panacea for Social Isolation

\author{
Diana Amundsen \\ Dr., University of Waikato, Tauranga, New Zealand \\ diana.amundsen@waikato.ac.nz
}

\begin{abstract}
This article explores whether digital communication technologies have applicability in reducing social isolation and loneliness among older adults. Issues of social isolation and loneliness among older adults are important as they are identified risk factors for mortality, disability, cognitive ability, depression and poor wellbeing. This problem is more urgent due to the COVID-19 pandemic which has required older adults to physically and socially distance from family, friends, neighbours, communities and health services. In the context of the present CoviD-19 pandemic, this article is of interest to educators, social workers, community service providers, health service practitioners, gerontological scholars involved in preparing older adult communities for present and future traumatic events resulting in socially isolating experiences. The literature identified that use of technology to promote social connection and enhance wellbeing for older adults can be an effective intervention, but more information is needed as to what aspects of such interventions make them effective. This research advocates for improvement in wellbeing and social connectedness of older adults through consideration of interventions through a model for flourishing and wellbeing. The research contributes to our growing understanding of how to change the way we think, feel and act towards older adults, ageing and flourishing.
\end{abstract}

\section{Keywords}

visual technologies against social isolation - older adults - social isolation loneliness - COVID-19 - digital technologies - ageism 


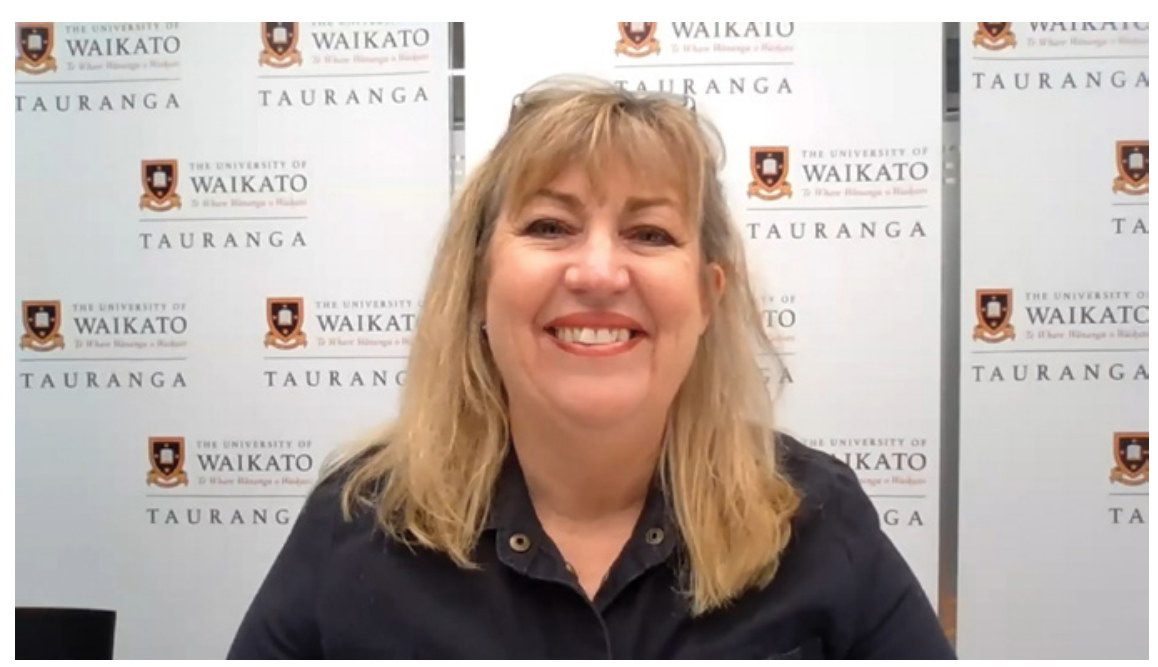

FEATURE Diana Amundsen's article comprises a video, which can be viewed here.

- This article is part of the special topic 'Visual Technologies as a Panacea for Social Isolation', edited by Rene Novak.

\section{Introduction}

\subsection{Pre-Pandemic Social Isolation and Loneliness}

Even before the COVID-19 pandemic, social isolation and loneliness were identified as significant health problems for older adults. Although often experienced together, social isolation and loneliness have slightly different meanings. Social isolation is an objective lack of relationships and social interaction, engagement and contacts, whereas, loneliness is a subjective, emotionally experienced distressing feeling and a perception of lack of meaningful relationships (Berg-Weger \& Morley, 2020; Coyle \& Dugan, 2012; Landeiro et al., 2017). Negative health outcomes connected with both social isolation and loneliness encompass depression, cardiovascular disease, quality of life and general poorer health (Courtin \& Knapp, 2015; Landeiro et al., 2017). Recent research claims that loneliness has similar health outcomes as smoking, obesity, lack of exercise and high blood pressure (Gott, 2020; Landeiro et al., 2017).

For these reasons and more, a growing body of literature draws attention to understanding and acting with intervention on the relationship between loneliness, social isolation and poor health and wellbeing among older adults (Antonucci et al., 2014; Gardiner et al., 2016; Petersen et al., 2016; Poscia, 2018). 
Various group-based and one-to-one interventions that may, or may not, be technology assisted have been developed to tackle this issue of reducing social isolation and loneliness. Examples include support groups, reminiscence therapy, videoconferencing, computer use training, animal companionship, educational courses on friendship, befriending volunteer programmes, home-delivered meals, internet and interactive games or activities and selfmanagement sessions (Landeiro et al., 2017). Yet, more methodologically sound and robust evidence of the scope of successful interventions and what aspects of such interventions make them effective is needed (Gardiner et al., 2016; Landeiro et al., 2017), particularly in understanding the individuality of the experience of loneliness. Furthermore, some traditional interventions for interacting with older adults have become impractical in the new normal context of COVID-19 where in-person activities may need to be suspended for long periods of time.

\subsection{COVID-19 Pandemic Effects of Social Isolation and Loneliness}

Since the COVID-19 pandemic, unprecedented attention has been given to the effects of social isolation and loneliness experienced by older adults. In part, this is due to the widespread finding across all nations that people older than 60 are clearly more susceptible to the COVID-19 disease and have thus far presented the highest mortality (Verity et al., 2020, Jiminez-Sotomayor et al., 2020). It may also be attributed to stringent containment strategies to protect older adults against infection, such as regional or national lockdowns, quarantine and physical distancing. While such measures might shield older adults from contracting the virus, the consequent effects of social isolation and poor selfmanagement of health due to reduced access to routine medical services have been pronounced (Chen, 2020). Older adults have experienced disruption in usual services that have prevented people from having their pre-existing physical, emotional and social needs met (Morrow-Howell et al., 2020).

Gaining an understanding of risk factors for social isolation and loneliness among older adults has become more critical in light of the Covid-19 pandemic. Berg-Weger and Morley (2020) identified risk factors as being related to, "living alone or in a rural community, poor functional status, widowhood, being female, lower income/education, losses, depression, and feeling misunderstood by others" (p. 456). Essentially, effects of the CoviD-19 pandemic have exacerbated these already existing inequalities of access to and participation in social connection and engagement activities. Steps to combat the spread and contraction of the disease through policies of social distancing have forced older adults to remain isolated at home or in facilities and have had a serious psychological toll, as was the case in previous pandemics (Roy et al., 2020). 
Anxiety, feelings of anger, emotional disturbances, stress, irritability, poor concentration, depression and inability to sleep were all psychological effects that emerged from the Middle Eastern Respiratory Syndrome (MERS) and the Severe Acute Respiratory Syndrome (sARs) pandemics (Berg-Weger \& Morley, 2020). In light of this, COVID-19 response efforts have swiftly proliferated to facilitate socio-psychological wellbeing and meaningful connection through the use of digital devices and visual communication technologies.

\section{Internet-based Technologies for Social Connection}

Digital technologies might be considered a panacea for older adults experiencing social isolation and loneliness both before and since the Covid-19 pandemic. In response to challenges of decreasing physical and social interactions and being 'locked up' or housebound, digital and visual technologies offer potential social connection means for older adults. Internet-based communication tools (for example, Skype, FaceTime, Zoom, WhatsApp, Messenger, and WeChat) offer low-cost or free opportunities to emotionally and socially connect while staying physically distanced. Chen (2020) states, "systematic reviews have revealed that internet- and mobile-based apps, companion robots, video games (Wii and TV gaming systems), video calls, and general computer usage are effective tools to reduce social isolation and loneliness for older adults..." (p. 12). In other words, maintaining existing relationships, gaining a sense of connection and social support and keeping up with hobbies or interests can be achieved through the use of digital and visual technologies (Chen, 2020; Poscia, 2018).

Opportunities to improve technology skills as well as family and intergenerational relationships have arisen through internet-based technologies used for social connection. Certainly, research is emerging about opportunities being adopted to improve the lives of older adults through increased connectivity (Morrow-Howell et al., 2020). It should be noted that numbers of tech-savvy older adults were on the rise prior to the pandemic (Nash, 2019) with growing numbers of people aged over 65 going online daily and owning a smartphone. When the pandemic arrived, setting in motion the quarantine and lockdown containment measures, a "sink or swim" moment arrived (Morrow-Howell et al., 2020, p. 530). It suddenly became urgent to order groceries online, conduct banking online, connect with family and friends through technology, and communicate with health-care providers, often online. For many older adults, this triggered an opportunity to motivate learning technology skills and usage for real purpose in lieu of regular face-to-face interactions. Wu et al. (2015) had 
already established that not being excluded from society was one main motivation for older adults to take up using internet technology, and this has been the case in the CoviD-19 pandemic. Improving uptake and use of internetbased digital technologies offers a means to reduce social isolation and loneliness through purposeful, regular and meaningful connections with peers, friends, family and community. News reports have also risen of increasing intergenerational communication and programmes where younger generations and cohorts interact with older adults, as seen in the following video clips (links under Figures 1 and 2).

Colenda et al. (2015) propose that intergenerational engagement is known to reduce ageism and bias. In this sense, intergenerational connections can

\section{Gen Z volunteers are helping senior citizens fight lockdown loneliness with technology}

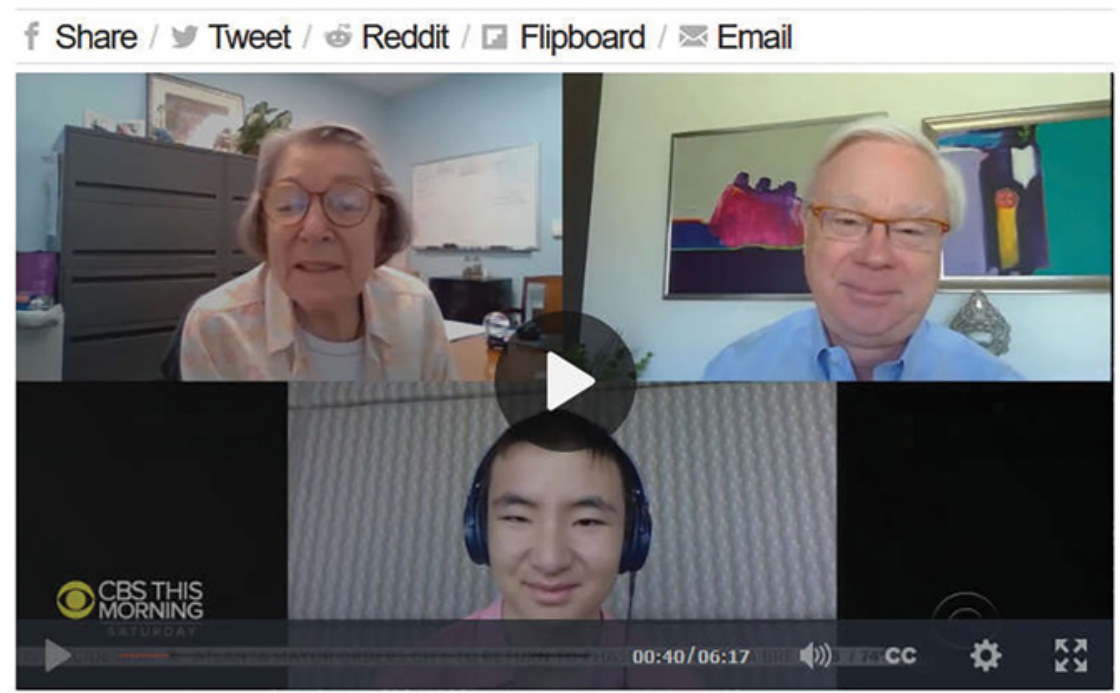

FIGURE 1 Gen Z volunteers are helping senior citizens fight lockdown loneliness with technology

HTTPS://WWW.CBSNEWS.COM/NEWS/GEN-Z-VOLUNTEERS-SENIOR-CITIZENS -LOCKDOWN-LONELINESS-TECHNOLOGY 


\section{Loneliness is on the rise due to the coronavirus. These entrepreneurs are making tackling isolation their business}

Published Tue, May 12 2020.11:34 PM EOT * Updated Wed, May 13 2020·10:09 AM EDT

Q Koren alechist

share $f y$ in $g$

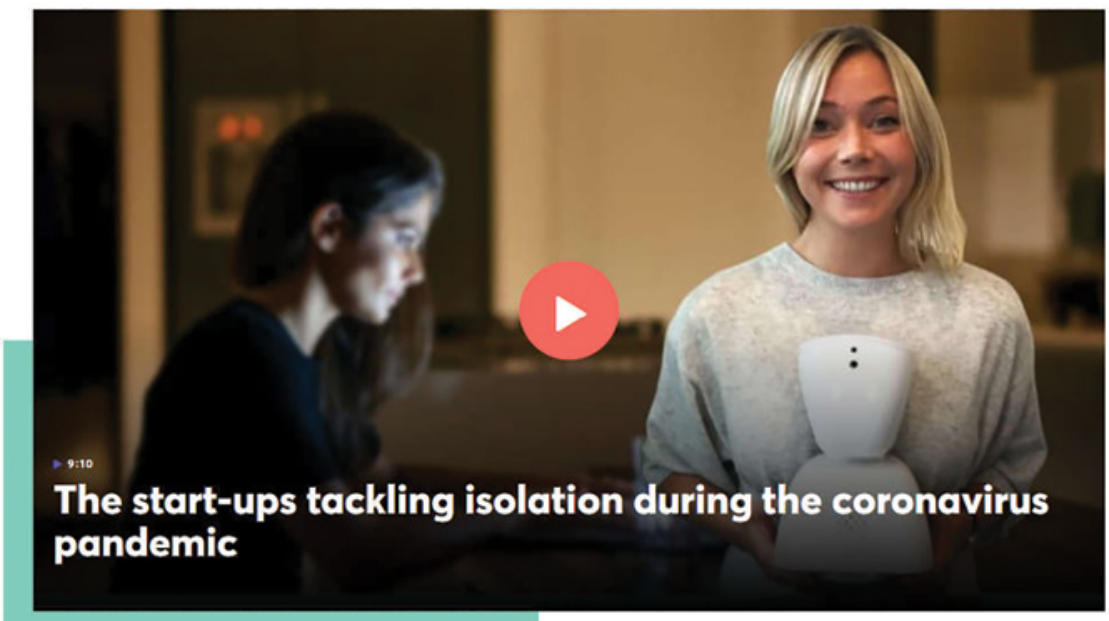

FIGURE 2 Loneliness is on the rise due to the coronavirus. These entrepreneurs are making tacking isolation their business

HTTPS://WWW.CNBC.COM/2020/05/13/ENTREPRENEURS-STARTUPS-ARE -TACKLING-CORONAVIRUS-ISOLATION-LONELINESS.HTML

have effects of reducing the perceived existence of a 'digital divide' (Wu et al., 2015) by engendering a deeper understanding of each other's generation and perspectives (Morrow-Howell et al., 2020).

\subsection{Barriers to Technology Use for Older Adults}

Older adults tend to face barriers and challenges in adopting internet-based technology when compared to younger 'technology native' generations for whom digital technology is widespread in schools, workplaces and homes. Certainly, digital visual communication technologies have offered a lifeline for many older adults staying at home during the pandemic. However, it would be a mistake to view older adults as a uniform, homogenous group. It cannot be ignored that barriers to accessing and using technology prevent many older adults from taking up these options. Further, some older adults experience a 
substantial transition when it comes to using computers and digital technology (Allis, 2010). For over-70-year olds, whose school life, work life and social life preceded the widespread use of digital technology of today, learning new skills in later life can present challenges. This is often further worsened by devices and software designed without inclusive principles in mind (e.g. large text, easy-to-read fonts, captions, small buttons, as found by Cisco, 2010). Furthermore, the typical physical decline that comes as part of the ageing process (Anton et al., 2015) can result in reduced manual dexterity to hold and control small devices (such as mobile phones). Additionally, older adults with hearing loss and impairments, impaired vision, and dementia can struggle to use digital devices not designed with their needs in mind.

Researchers have begun exploring typical barriers faced by older adults when using digital technology. Financial cost of purchasing devices and maintaining monthly internet access is a common barrier (Berry, 2011). Other barriers found in the literature include inappropriate design, lack of experience, low awareness, concerned attitude or lack of interest, lack of general knowledge of technology products and services, lack of support or help, being nervous or afraid, do not see personal benefits to themselves, unhappy about what they hear about dangers of technology use and more (see Dellelo \& McWhorter, 2015 for a comprehensive literature review). Taken together, all these barriers amount to a significant difference in ability to participate in society and build meaningful relationships and social connections. This difference has often been referred to as part of a wider, well-known phenomenon called the 'digital divide' (Compaine, 2001).

\subsection{Digital Divide of Technology Access and Use with Older Adults}

Digital divide can no longer be conceived of as a single divide expressed in binary terms between those with access to technology and those without. Older adults with a lower socio-economic status are at increased risk for social, economic and physical difficulties brought on by the pandemic. For example, internet-based communication has eased social isolation for many older adults, but for those with lower income, access is challenging, if not negligible (Morrow-Howell et al., 2020). Wu et al. (2015) state, "a digital divide is commonly considered as social inequality and social injustice that a society must find ways to bridge" (p. 199). Hill et al. (2015) found that inclusion and exclusion of digital technology usage relates to social and systemic issues at both micro and macro levels. For instance, at the micro level, frustration, physical and mental limitations mistrust, time issues and self-efficacy (Gatto \& Tak, 2008) impact on individual older adults' likelihood to adopt technologies. Implications at the macro level indicate that organisational and societal policies need 
to confront barriers for older adults' digital technology usage such as addressing fears related to security of technology and personal data. Moreover, social and systemic policies regarding older adults need careful scrutiny to ensure the avoidance of ageist language, ideas and strategies.

\section{$3 \quad$ Ageism}

Fundamentally, ageism concerns the prejudices held by one group of people towards another group of people because of their age (Henry et al., 2019). Even before the COVID-19 pandemic, the system of stereotypes, biases and discrimination experienced by older adults due to age were a growing concern (World Health Organisation, 2017). Three forms of ageism have been identified: limitations of activities or opportunities; cultural beliefs that social, psychological and individual experiences are defined by age; misplaced belief that results of studies of one age-group can be generalized to other age groups (Henry et al., 2019). Ageism can have profoundly negative effects for older adults in many domains of life, for instance in employment, healthcare, media portrayal, everyday life and conversation (Henry et al., 2019). Therefore ageism can influence beliefs, thoughts, and actions concerning older adults and have profoundly negative impacts within individual's lives.

\subsection{Ageism Intensified through coVID-19}

Ageism in association with the COVID-19 pandemic has been intensified through the emergence of an ageist public discourse positioning older adults as vulnerable, weak, inferior, less capable of adaptation to difficulties (e.g. adopting technology), less resilient, and a burden (Colenda et al., 2020). Pertinent examples are seen in new terms such as "boomer remover" (Aronson, 2020), "thinning the herd" (Morrow-Howell, 2020) and jokes or ridicule of older adults circulating on social media (Jiminez-Sotomayor et al., 2020). Colenda et al. (2020) note, "Society's diminished valuation of individuals as they age is a continuing thread within ageism. Tackling ageism in this pandemic requires evidence that contravenes these preconceived notions" (p. 1627). Professor Merryn Gott, one of Aotearoa New Zealand's leading researchers of older adults, proposes that ignoring the vital contribution older adults make to their communities is unhelpful and ill-considered (Gott, 2020). Importantly, more older adults should be included in policies and decision-making solutions. Seeking the voices of diverse older adults to understand their perspectives on social connection is one way to tackle ageist notions. This short animation 


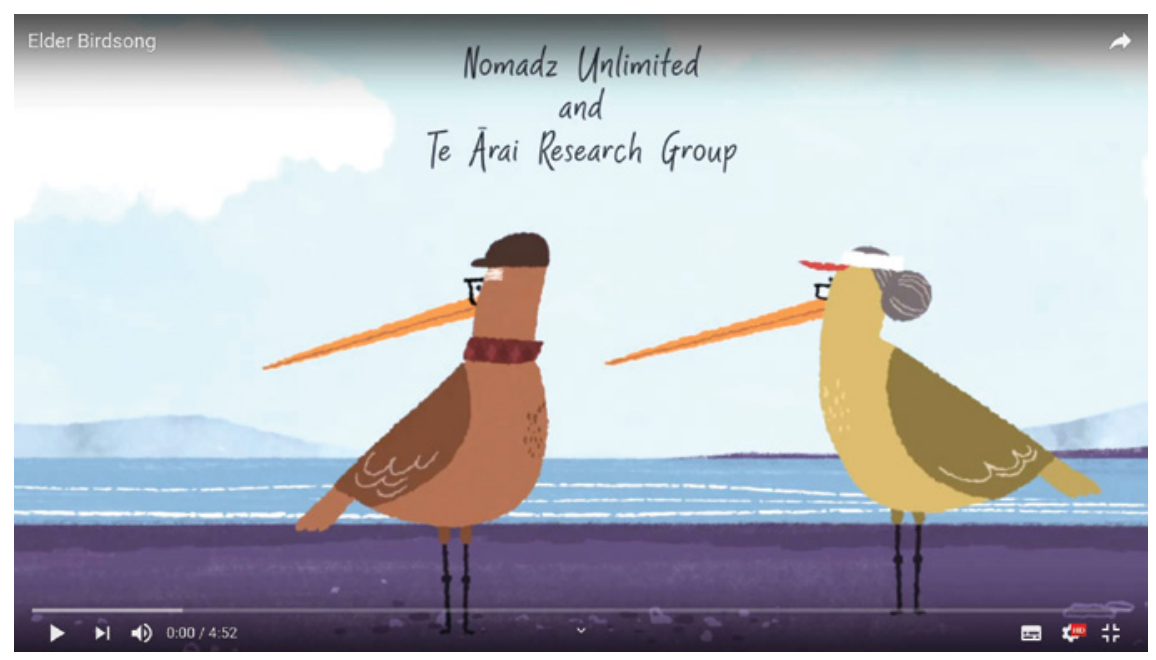

FIGURE 3 Elder birdsong

HTTPS://WWW.YOUTUBE.COM/WATCH?RELOAD=9\&V=OWFRKDWCICY

video was designed to give a window into the diverse voices of older adults in Aotearoa who face social isolation

The COVID-19 pandemic has exposed long-held ageist attitudes and actions being externally expressed in society. For instance, Morrow-Howell et al. (2020) found that some countries were slower to respond to the pandemic because they perceived that it was only older people who were at risk. Such actions and attitudes may also serve to strengthen internalized ageism-some older adults resist identifying themselves as at-risk because it means acknowledging the reality of their age (Aronson, 2020). Internalized ageism, taken with external stereotyping and discrimination of older adults have been further exacerbated through this pandemic.

\subsection{Age as a Social Construct}

Ageism can be linked to the social construction of age. Conceptually, theoretical foundations of this article position the study of age as a social construct. Ageing is not just a natural, inevitable process, but is largely influenced and determined by sociocultural factors (Andrew, 2012). Since Philippe Ariès (1962) first introduced his seminal work Centuries of Childhood depicting the social construction of childhood, perspectives of ageing more generally as a social construction have become widespread (Andrew, 2012). A socially constructed perspective views ageing as a discursive process whereby people imbue meaning to the experience of ageing; individuals develop their age identity 
by social interactions (Andrew, 2012). The complexity of age identity, from a poststructuralist perspective views the concept of age as multiple, fragmented, changeable and a site of struggle. Although this article has highlighted some of the consequences of social disconnection experienced by older adults, the pandemic represents an opportunity for anti-ageism activists to promote rethinking of societal commitments to valuing and respecting older adults. By throwing a spotlight on the inequalities experienced by older adults during the pandemic crisis, a more robust social justice and wellness equity voice has gained some ground in the site of ageism struggles (Morrow-Howell et al., 2020).

\subsection{Positivity and Flourishing in Ageing}

This research aims to advocate for improvement in the wellbeing and social connectedness of older adults. Through understanding how stereotyping, prejudice and discrimination against older adults on the basis of their age has profoundly negative consequences, the research contributes to our growing understanding of how to change the way we think, feel and act towards age, ageing and flourishing. The literature reviewed earlier identifies that use of technology to promote social connection and enhance wellbeing for older adults can be an effective intervention. More robust information is needed as to what aspects of such interventions make them effective, and how to reduce barriers for access and participation in technology usage.

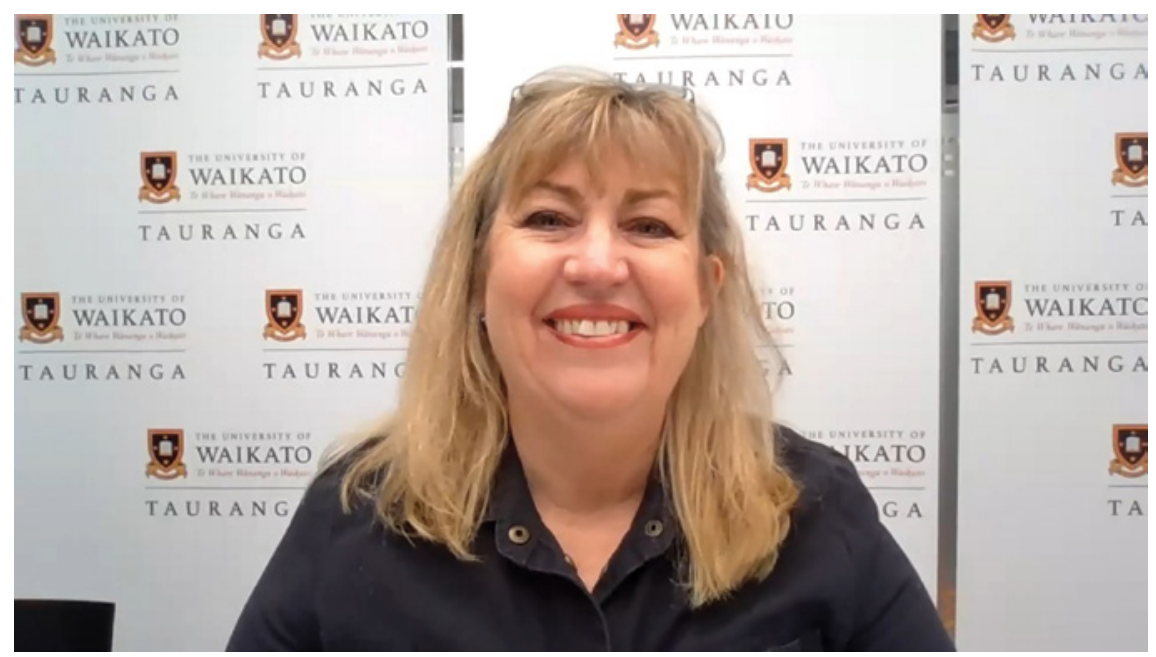

VIDEo 1 Diana's podcast: the Wellbeing and Positivity Model in Ageing. (See here.) 
One model that has potential for guiding the development, measurement and ongoing sustainability for technology interventions to enhance the wellbeing of older adults is the 'Flourishing Protocol' (Renaissance Coaching, 2017). Based on an evidence-informed positive psychology framework of five domains originally conceived by Seligman (2011) and subsequently added to by Emiliya Zhivotovskaya (O'Brien, 2014), the flourishing protocol comprises six psycho-social and physical domains of positivity, engagement, relationships, meaning, achievement and vitality, as further explained in Video 1.

The flourishing model presents a lens through which to view the creation, evaluation and effectiveness of technology use to resolve social isolation and loneliness in older adults' lives.

\section{4}

\section{Conclusion}

This article contributes to our growing understanding of how to change the way we think, feel and act towards age, ageing and flourishing. In 2017, the United States Surgeon General Vivek Murthy (2017) commanded international attention by declaring there was a "global loneliness epidemic" to describe the social isolation and loneliness in the world's older adult population. The COVID-19 pandemic has further intensified very real and sad situations for many older adults throughout the world.

Simultaneously, the pandemic has presented an unexpected and exceptional opportunity to address the loneliness epidemic through the development and adaptation of internet-based technology for social connections. This research calls for the growth of robust evidence-based interventions and presents the flourishing protocol as a means to develop, measure and sustain digital technology-based interventions for positive health and wellbeing for older adults. Not only do flourishing individuals thrive, they are also filled with socio-psycho vitality both in their private and social lives. We have an opportunity to reconstruct attitudes, beliefs and actions towards older adults by reconceiving our notions of what it means to age well.

\section{References}

Allis, S. (2010). For elderly, wired world holds terror or delight. Boston Globe. Retrieved from: https://www.boston.com/news/local/massachusetts/articles/2010/04/06/for _elderly_the_wired_world_holds_terror_or_delight. 
Anton, S. D., Woods, A. J., Ashizawa, T., Barb, D., Buford, T. W., Carter, C. S., ... Pahor, M. (2015). Successful aging: Advancing the science of physical independence in older adults. Ageing research reviews, 24(Pt B), 304-327. https://www.doi.org/10.1016/j .arr.2015.09.005.

Antonucci, T. C., Ajrouch, K. J., Birditt, K. S. (2014). The convoy model: Explaining social relations from a multidisciplinary perspective. Gerontologist, 54, 82-92. doi:10.1093/ geront/gnt118.

Andrew, P. (2012). The Social Construction of Age: Adult Foreign Language Learners. UK: Channel View Publications.

Ariès, P. (1962). Centuries of childhood; a social history of family life. New York: Knopf.

Aronson, L. (2020). Ageism is making the pandemic worse. The Atlantic. https://www .theatlantic.com/culture/archive/2020/03/americas-ageism-crisis-is-helping-the -coronavirus/608905.

Berg-Weger, Marla, \& Morley, J. E. (2020). Loneliness and Social Isolation in Older Adults during the Covid-19 Pandemic: Implications for Gerontological Social Work. The Journal of Nutrition, Health \& Aging, 24(5), 456-458. https://www.doi .org/10.1007/s12603-020-1366-8.

Chen, K. (2020). Use of gerontology to assist older adults to cope with the CoviD-19 pandemic. Journhal of the American Medical Directors Association, 21(7), 983-984. https://www.doi.org/10.1016/j.jamda.2020.05.021.

Cisco. (2010). Older people, technology and community. Independent Age. Retrieved from https://www.cisco.com/web/about/ac79/docs/wp/ps/Report.pdf.

Colenda, C., Reynolds, C., Applegate, W., Sloane, P., \& Zimmerman, S. (2020). Covid-19 Pandemic and Ageism: A call for humanitarian care. American Journal of Geriatric Psychiatry, 28(8), 805-807. doi:10.1016/j.jagp.2020.04.005.

Compaine, B. (2001). The digital divide: Facing a crisis or creating a myth? Cambridge, Massachusetts: MIT Press.

Courtin, E., \& Knapp, M. (2015). Social isolation, loneliness and health in old age: a scoping review. Health and Social Care in the Community. doi:10.1111/hsc.12311.

Coyle, C., \& Dugan, E. (2012)., Social isolation, loneliness and health among older adults. https://www.doi.org/10.1177/0898264312460275.

Delello, J., \& McWhorter, R. (2017). Reducing the Digital Divide: Connecting Older Adults to iPad Technology. Journal of Applied Gerontology, 36(1). doi:10.1177/ 0733464815589985 .

Gatto, S., \& Tak, S. (2008). Computer, Internet, and E-mail Use Among Older Adults: Benefits and Barriers. Educational Gerontology, 34 (9), 80o-811. https://www.doi .org/10.1080/03601270802243697.

Gardiner, C., Geldenhuys, G., \& Gott, M. (2016). Interventions to reduce social isolation and loneliness among older people: an integrative review. https://www.doi .org/10.1111/hsc.12367. 
Gott, M. (2020a). Talking to - not at - older Kiwis. 3 July 2020. Newsroom. https://www .newsroom.co.nz/ideasroom/auckland-oped-on-old-peoplelockdown.

Gott, M. (202ob). Social connectedness among older people during COVID-19. https:// www.medicalresearch.org.nz/post/social-connectedness-among-older-people -during-covid-19.

Henry, R., Perrin, P., \& Smith, E. (2019). The Underpinnings of Ageism: Multiple Mediational Model of Epistemological Style, Social Dominance Orientation, Right-Wing Authoritarianism, and Ageist Attitudes. Journal of Aging Research, 2019, 1-8. https:// www.doi.org/10.1155/2019/3672725.

Hill, R., Betts, L., Gardner, L. (2015). Older adults' experiences and perceptions of digital technology: (Dis)empowerment, wellbeing, and inclusion. https://www.doi .org/10.1016/j.chb.2015.01.062.

Jimenez-Sotomayor, M., Gomez-Moreno, C., \& Soto-Perez-de-Celis, E. (2020). Coronavirus, Ageism, and Twitter: An evaluation of tweets about older adults and Covid-19. American Geriatric Society, 68(8), 1661-1665. doi: 10.1111/jgs.16508.

Landeiro, F., Barrows, P., Ellen, N. M., Gray, A. M., \& Leal, J. (2017). Reducing social isolation and loneliness in older people: A systematic review protocol. BMJ Open, 7(5). https://www.doi.org.ezproxy.waikato.ac.nz/10.1136/bmjopen-2016-013778.

Morrow-Howell, N., Galucia, N., Swinford, E. (2020). Recovering from the covid-19 Pandemic: A focus on older adults. Journal of Aging \& Social Policy, 32 (4-5), 526535. https://www.doi.org/10.1080/08959420.2020.1759758.

MurthyV.(2017).Work and the loneliness epidemic.Harvard Business Review, September 2017. Retrieved from: https://www.vivekmurthy.com/single-post/2017/10/10/Work -and-the-Loneliness-Epidemic-Harvard-Business-Review.

Nash, S. (2019, April 13). Older adults and technology: Moving beyond the stereotypes. Stanford Center of Longevity. Retrieved from: https://longevity.stanford. edu/2019/05/30/older-adults-and-technology-moving-beyond-the-stereotypes.

O’Brien, E. (2014, November 19). PERMA-v: Training with rigor \& vigor. Retrieved from https://www.positivepsychologynews.com/news/elaine-obrien/2014111930383.

Poscia, A., Stojanovic, J., La Milia, D. (2018). Interventions targeting loneliness and social isolation among the older people: An update systematic review. Exp Gerontolog., 102, 133-144. doi:10.1016/j.exger.2017.11.017.

Petersen, J., Kaye, J., Jacobs, P., Quinones, A., Dodge, H., Arnold, A, \& Thielke, S. (2016). Longitudinal Relationship Between Loneliness and Social Isolation in Older Adults. Journal of Aging and Health, 28(5), 775-795. https://www.doi.org/10.1177/ 0898264315611664 .

Roy, J., Jain, R., Golamari, R., Vunnam, R., Sahu, N. (2020). Covid-19 in the geriatric population. International Journal of Geriatric Psychiatry. https://www.doi.org/10.1002/ gps.5389.

Seligman, M. (2011). Flourish. New York, NY: Simon \& Schuster. 
Verity, R., Okell, L., Dorigatti, I. (2020). Estimates of the severity of coronavirus disease 2019: A model-based analysis. Lancet Infect Dis, 20, 669-677. https://www.doi .org/10.1016/S1473-3099(20)30243-7.

World Health Organisation (2017). Global strategy and action plan on ageing and health. Retrieved from: https://www.who.int/ageing/WHO-GSAP-2017.pdf?ua=1. 